\title{
A choice of bilevel linear programming solving parameters: factoraggregation approach
}

\author{
Pavels Orlovs $^{1}$ Olga Montvida ${ }^{1}$ Svetlana Asmuss $^{1,2}$ \\ ${ }^{1}$ Department of Mathematics, University of Latvia \\ ${ }^{2}$ Institute of Mathematics and Computer Science, University of Latvia
}

\begin{abstract}
Our paper deals with the problem of choosing correct parameters for the bilevel linear programming solving algorithm proposed by M. Sakawa and I. Nishizaki. We suggest an approach based on factoraggregation, which is a specially designed general aggregation operator. The idea of factoraggregation arises from factorization by the equivalence relation generated by the upper level objective function. We prove several important properties of the factoraggregation result regarding the analysis of parameters in order to find an optimal solution for the problem. We illustrate the proposed method with some numerical and graphical examples, in particular we consider a modification of the mixed production planning problem.
\end{abstract}

Keywords: General aggregation operator, multiobjective linear programming problem, bilevel linear programming problem

\section{Introduction}

The paper is devoted to bilevel linear programming problems (BLPP) with only one objective on the upper level. We propose a special design of aggregation operator for the analysis of the satisfaction degree of objectives on each level and the choice of solving parameters values. In the context of our research each objective generates a fuzzy set corresponding to the objective membership function. As a result, the proposed aggregation operator is a general aggregation operator. We investigate its properties and describe an application scheme.

In the first section we consider formulation of BLPP, which is a special class of multi-objective linear programming problems (MOLP). Then we observe the MOLP fuzzy solution method involving membership functions of objectives introduced by H.J. Zimmermann [10]. Using this method the initial MOLP can be reduced to the ordinary linear programming problem, where an aggregation of all objectives' membership functions is maximized. In the second section we recall the definitions of an ordinary aggregation operator and of a general aggregation operator acting on fuzzy structures, these concepts were introduced by A. Takaci [9]. Then we introduce a construction of a factoraggregation operator, which is a case of the general aggregation operator. The idea of factoraggregation is based on factorization by an equivalence relation. We show that all properties of the definition of a general aggregation operator such as the boundary conditions and the monotonicity hold for the factoraggregation operator. In the third section we show how factoraggregation can be applied for solving BLPP. This section is based on the interactive method of solution of bilevel linear programming problems introduced by M. Sakawa and I. Nishizaki [7],[8] and involving some parameters for the upper and lower level objectives. We illustrate with some numerical and graphical examples showing how factoraggregation is applied to the analysis of the choice of the parameters for solving BLPP. Several important properties of the result of factoraggregation are proved, these properties help us in the process of choosing the solving parameters. In the final section we illustrate the factoraggregation approach with the analysis of solving parameters for one particular problem called the mixed production planning problem. We modify the problem described in [3] by considering new objective functions: we maximize the profit of a production company with the higher priority and minimize the volume of environmentally damaging products and the dependence of external suppliers. We give numerical values for the parameters of the problem and describe how the analysis of solving parameters could be performed.

\section{BLPP fuzzy solution approach}

In this paper we observe a bilevel linear programming problem with one objective on the upper level $P^{U}$ with the higher priority in optimization than multiple objectives on the lower level $P^{L}=$ $\left(P_{1}^{L}, P_{2}^{L}, \ldots, P_{n}^{L}\right)$ :

$P^{U}: \quad y_{0}(x)=c_{01} x_{1}+c_{02} x_{2}+\ldots+c_{0 k} x_{k} \longrightarrow \min$

$P_{1}^{L}: \quad y_{1}(x)=c_{11} x_{1}+c_{12} x_{2}+\ldots+c_{1 k} x_{k} \longrightarrow \min$

$P_{n}^{L}: y_{n}(x)=c_{n 1} x_{1}+c_{n 2} x_{2}+\ldots+c_{n k} x_{k} \longrightarrow \min$

$D:\left\{\begin{array}{l}a_{j 1} x_{1}+a_{j 2} x_{2}+\ldots+a_{j k} x_{k} \leq b_{j}, j=\overline{1, m}, \\ x_{l} \geq 0, l=\overline{1, k}\end{array}\right.$

where $k, l, m, n \in \mathbb{N}, a_{j l}, b_{j}, c_{i l} \in \mathbb{R}, j=\overline{1, m}$, $l=\overline{1, k}, i=\overline{0, n}$, and $x=\left(x_{1}, \ldots, x_{k}\right) \in \mathbb{R}^{k}$. We 
assume that $D \subset \mathbb{R}^{k}$ is non-empty and bounded, and we consider the case when the constraint $x \in D$ is related to all levels.

As all objectives rarely reach their optimal values in a single point, a compromise solution should be found. In multi-objective optimization Pareto optimality (see, e.g., [5]) is a concept that allows us to characterize an acceptable solution.

Definition 2.1 $x^{*} \in D$ is said to be a Pareto optimal solution if and only if there does not exist another $x \in D$ such that $y_{i}(x) \leq y_{i}\left(x^{*}\right)$ for all $i=\overline{0, n}$ and $y_{j}(x) \neq y_{j}\left(x^{*}\right)$ for at least one $j$.

In 1978 H.J. Zimmermann [10] proposed a fuzzy solution approach by introducing membership functions of objectives. The membership function characterises the degree of satisfaction for each objective, i.e. it shows how the objective function is close to its optimal value (i.e. to its individual maximum). The construction of the membership function of objective $y_{i}$ is based on the following function:

$$
z_{i}(t)= \begin{cases}1, & t<y_{i}^{\min } \\ \frac{t-y_{i}^{\max }}{y_{i}^{\min }-y_{i}^{\max }}, & y_{i}^{\text {min }} \leq t \leq y_{i}^{\max } \\ 0, & t>y_{i}^{\max }\end{cases}
$$

where $y_{i}^{\min }$ and $y_{i}^{\max }$ are the individual minimum and the individual maximum of the objective $y_{i}$ respectively:

$$
y_{i}^{\min }=\min _{x \in D} y_{i}(x), y_{i}^{\max }=\max _{x \in D} y_{i}(x), \quad i=\overline{0, n} .
$$

We obtain the membership functions of the objectives by denoting

$$
\mu_{i}(x)=z_{i}\left(y_{i}(x)\right), i=\overline{0, n}
$$

where $\mu_{0}, \mu_{1}, \ldots, \mu_{n}: D \rightarrow[0,1]$ are fuzzy subsets of $D$ :

$$
\mu_{i} \in[0,1]^{D}, i=\overline{0, n} .
$$

A solution $x^{*}$ for the MOLP without any hierarchy could be found by solving the following linear programming problem:

$$
\min \left(\mu_{0}(x), \mu_{1}(x), \ldots, \mu_{n}(x)\right) \longrightarrow \max _{x \in D}
$$

or, in general:

$$
A\left(\mu_{0}(x), \mu_{1}(x), \ldots, \mu_{n}(x)\right) \longrightarrow \max _{x \in D}
$$

where $A$ is an aggregation operator (see Definition 3.1 . However, in case when the objectives are divided between two levels of hierarchy, the present method does not reflect any priority of the upper level objective over the lower level.

\section{Factoraggregation}

Considering the case when there is one objective function on the upper level and multiple objectives on the lower level, we suggest a special aggregation. This aggregation observes objective functions on the lower level considering the classes of equivalence generated by a function on the upper level:

$$
\begin{gathered}
\tilde{A}_{\mu_{0}}\left(\mu_{1}, \mu_{2}, \ldots, \mu_{n}\right)(x)= \\
=\max _{\mu_{0}(x)=\mu_{0}(u)} A\left(\mu_{1}(u), \mu_{2}(u), \ldots, \mu_{n}(u)\right),
\end{gathered}
$$

where

$$
x, u \in D, \quad \mu_{0}, \mu_{1}, \ldots, \mu_{n} \in[0,1]^{D} .
$$

Aggregation $\tilde{A}_{\mu_{0}}$ is a specially designed construction of a general aggregation operator based on an ordinary aggregation operator $A$. As the examples of widely used aggregation operators we can mention the arithmetic and geometric means, the minimum and maximum operators, $t$-norms and others. We start with the classical notion of an aggregation operator (see e.g. [1], [2], [4]).

Definition 3.1 A mapping $A: \bigcup_{n}[0,1]^{n} \rightarrow[0,1]$ $i s$ called an aggregation operator if the following conditions hold:

$$
\begin{aligned}
& A(0, \ldots, 0)=0 \\
& \quad A(1, \ldots, 1)=1 ; \\
& \forall n \in \mathbb{N} \forall x_{1}, \ldots, x_{n}, y_{1}, \ldots, y_{n} \in[0,1]: \\
& \text { if } x_{1} \leq y_{1}, \ldots, x_{n} \leq y_{n}, \text { then } A\left(x_{1}, \ldots, x_{n}\right) \leq \\
& A\left(y_{1}, \ldots, y_{n}\right) .
\end{aligned}
$$

Conditions (A1) and (A2) are called the boundary conditions of $A$, but (A3) means the monotonicity of $A$.

The general aggregation operator $\tilde{A}$ acting on $[0,1]^{D}$, where $[0,1]^{D}$ is the set of all fuzzy subsets of $D$, was introduced in 2003 by A. Takaci [9]. Let $\mu_{1}, \mu_{2}, \ldots, \mu_{n} \in[0,1]^{D}$ be fuzzy sets. We denote an order on $[0,1]^{D}$ by $\preceq$, the least and the greatest elements of this order are denoted by $\tilde{0}$ and $\tilde{1}$, which are indicators of $\varnothing$ and $D$ respectively, i.e.

$$
\tilde{0}(x)=0 \text { and } \tilde{1}(x)=1 \text { for all } x \in D .
$$

Definition 3.2 A mapping $\tilde{A}: \bigcup_{n}\left([0,1]^{D}\right)^{n} \rightarrow$ $[0,1]^{D}$ is called a general aggregation operator if the following conditions hold:

$$
\begin{aligned}
& \tilde{A}(\tilde{0}, \ldots, \tilde{0})=\tilde{0} \\
& \tilde{A}(\tilde{1}, \ldots, \tilde{1})=\tilde{1} ; \\
& \forall n \in \mathbb{N} \forall \mu_{1}, \ldots, \mu_{n}, \eta_{1}, \ldots, \eta_{n} \in[0,1]^{D}: \\
& \text { if } \mu_{1} \preceq \eta_{1}, \ldots, \mu_{n} \preceq \eta_{n}, \text { then } \tilde{A}\left(\mu_{1}, \ldots, \mu_{n}\right) \preceq \\
& \tilde{A}\left(\eta_{1}, \ldots, \eta_{n}\right) .
\end{aligned}
$$

There exist several approaches to construct a general aggregation operator $\tilde{A}$ based on an ordinary aggregation operator $A$. The most simplest one is 
the pointwise extension of an aggregation operator $A$ :

$$
\tilde{A}\left(\mu_{1}, \ldots, \mu_{n}\right)(x)=A\left(\mu_{1}(x), \ldots, \mu_{n}(x)\right),
$$

which, for example, was already used in the previous section.

Another method of constructing the general aggregation operator $\tilde{A}$ is the $t$ - extension [9], whose idea comes from the classical extension principle and uses a $t$-norm (see e.g. [6]).

We introduce a factoraggregation of fuzzy sets $\mu_{1}, \mu_{2}, \ldots, \mu_{n}$ by means of a fuzzy set $\mu_{0}$ using the following construction:

$$
\begin{gathered}
\tilde{A}_{\mu_{0}}\left(\mu_{1}, \ldots, \mu_{n}\right)(x)= \\
=\sup _{\mu_{0}(u)=\mu_{0}(x)} A\left(\mu_{1}(u), \ldots, \mu_{n}(u)\right),
\end{gathered}
$$

where

$$
\mu_{0}, \ldots, \mu_{n} \in[0,1]^{D}, x, u \in D
$$

The motivation of using the term factoraggregation for $\tilde{A}_{\mu_{0}}$ is that $\mu_{0}$ generates the equivalence relation $\sim_{\mu_{0}}$ :

$$
u \sim_{\mu_{0}} v \Longleftrightarrow \mu_{0}(u)=\mu_{0}(v),
$$

which factorizes $D$ into the classes $D^{\alpha}$ of equivalence:

$$
D^{\alpha}=\left\{x \in D \mid \mu_{0}(x)=\alpha\right\} .
$$

Operator $\tilde{A}_{\mu_{0}}$ aggregates fuzzy sets in accordance with these classes of equivalence.

Let us show that properties $(\tilde{A} 1)-(\tilde{A} 3)$ hold for operator $\tilde{A}_{\mu_{0}}$, therefore we can be sure that $\tilde{A}_{\mu_{0}}$ is a general aggregation operator. We consider the case:

$\mu \preceq \eta$ if and only if $\mu(x) \leq \eta(x)$ for all $x \in D$.

Proposition 3.3 Operator $\tilde{A}_{\mu_{0}}$ is a general aggregation operator.

Proof. First we prove the boundary conditions:

1)

$$
\begin{gathered}
\tilde{A}_{\mu_{0}}(\tilde{0}, \ldots, \tilde{0})(x)= \\
=\sup _{\mu_{0}(u)=\mu_{0}(x)} A(\tilde{0}(u), \ldots, \tilde{0}(u))= \\
=\sup _{\mu_{0}(u)=\mu_{0}(x)} A(0, \ldots, 0)=A(0, \ldots, 0)=\tilde{0}(x),
\end{gathered}
$$

2)

$$
\begin{gathered}
\tilde{A}_{\mu_{0}}(\tilde{1}, \ldots, \tilde{1})(x)= \\
=\sup _{\mu_{0}(u)=\mu_{0}(x)} A(\tilde{1}(u), \ldots, \tilde{1}(u))= \\
=\sup _{\mu_{0}(u)=\mu_{0}(x)} A(1, \ldots, 1)=A(1, \ldots, 1)=\tilde{1}(x) .
\end{gathered}
$$

To prove the monotonicity of $\tilde{A}_{\mu_{0}}$ we use the monotonicity of $A$ :

$$
\begin{gathered}
\mu_{i} \preceq \eta_{i}, \quad i=1,2, \ldots, n \Longrightarrow \\
\Longrightarrow A\left(\mu_{1}(u), \ldots, \mu_{n}(u)\right) \leq \\
\leq A\left(\eta_{1}(u), \ldots, \eta_{n}(u)\right) \text { for all } u \in D \Longrightarrow \\
\Longrightarrow \sup _{\mu_{0}(u)=\mu_{0}(x)} A\left(\mu_{1}(u), \ldots, \mu_{n}(u)\right) \leq \\
\leq \sup _{\mu_{0}(u)=\mu_{0}(x)} A\left(\eta_{1}(u), \ldots, \eta_{n}(u)\right) \Longrightarrow \\
\Longrightarrow \tilde{A}_{\mu_{0}}\left(\mu_{1}, \ldots, \mu_{n}\right) \preceq \tilde{A}_{\mu_{0}}\left(\eta_{1}, \ldots, \eta_{n}\right) .
\end{gathered}
$$

\section{Factoraggregation applied for analysis of BLPP solving parameters}

By using membership functions $\mu_{0}, \mu_{1}, \ldots, \mu_{n}$ the multi-objective linear programming problem can be reduced to the classical linear programming (LPP):

$$
\begin{gathered}
\sigma \longrightarrow \max _{x, \sigma} \\
\left\{\begin{array}{l}
\mu_{i}(x) \geq \sigma, \quad i=\overline{0, n}, \\
x \in D,
\end{array}\right.
\end{gathered}
$$

which is equivalent to the following problem:

$$
\min \left(\mu_{0}(x), \mu_{1}(x), \ldots, \mu_{n}(x)\right) \longrightarrow \max _{x \in D}
$$

(here we use the additional real variable $\sigma$ ). Let us denote the solution of this LPP by $\left(x^{*}, \sigma^{*}\right)$. In [5] there is described how to verify whether $x^{*}$ is Pareto optimal.

For bilevel linear programming problems M. Sakawa and I. Nishizaki [7],[8] proposed the interactive method of solution by involving some parameters for the upper and lower level objectives. The algorithm specifies an optimal solution $x^{* *}$ for BLPP according to the chosen values of real parameters $\delta, \Delta_{L}, \Delta_{U}$, where

$$
\begin{gathered}
\mu_{0}\left(x^{* *}\right) \geq \delta \\
\Delta_{L} \leq \Delta=\frac{\min \left\{\mu_{1}\left(x^{* *}\right), \ldots, \mu_{n}\left(x^{* *}\right)\right\}}{\mu_{0}\left(x^{* *}\right)} \leq \Delta_{U} .
\end{gathered}
$$

By this method (see [7],[8]) we solve the linear programming problem

$$
\sigma \longrightarrow \max _{x, \sigma}
$$

$$
\left\{\begin{array}{l}
\mu_{0} \geq \delta, \\
\mu_{i}(x) \geq \sigma, \quad i=\overline{1, n}, \\
x \in D,
\end{array}\right.
$$

for a given $\delta$, afterwards we check whether $\Delta \in$ $\left[\Delta_{L}, \Delta_{U}\right]$ and specify the parameters again if it is necessary. 
Parameter $\delta$ describes the minimal satisfactory level for membership function $\mu_{0}$, but $\Delta$ characterizes the overall balance between the upper and lower levels. Taking into account that all three parameters are dependent one on another, the problem of the choice of parameters becomes important. Let us consider the following BLPP.

\section{Example 4.1}

$$
\begin{gathered}
P^{U}: \quad y_{0}(x)=-x_{2} \longrightarrow \min \\
P_{1}^{L}: \quad y_{1}(x)=-3 \sqrt{3} x_{1}+3 x_{2} \longrightarrow \min \\
P_{2}^{L}: \quad y_{2}(x)=3 \sqrt{3} x_{1}+3 x_{2} \longrightarrow \min \\
D:\left\{\begin{array}{l}
3 \sqrt{3} x_{1}+3 x_{2} \leq 18 \sqrt{3}, \\
-3 \sqrt{3} x_{1}+3 x_{2} \leq 0, \\
x_{l} \geq 0, x_{2} \geq 0 .
\end{array}\right.
\end{gathered}
$$

Fig. 1 shows how parameter $\delta$ depends on parameter $\Delta$. On Fig. 1 we can see, that if we choose parameter $\delta=0.6$ and interval $\left[\Delta_{L}, \Delta_{U}\right]=[0.6,0.7]$, then a solution for the problem doesn't exist. If we first choose the interval $\left[\Delta_{L}, \Delta_{U}\right]=[0.6,0.7]$, then the maximal possible value for $\delta$ is 0.46 . But in case, when the value of $\delta$ is 0.6 , then the maximal possible value of $\Delta$ is 0.33 .

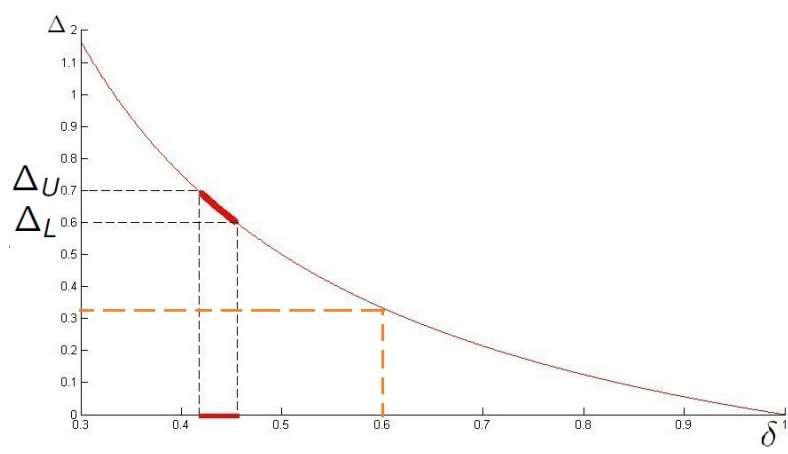

Figure 1: Dependence between $\delta$ and $\Delta$ for Example 4.1

We specify the general construction of factoraggregation $\tilde{A}_{\mu_{0}}$ by taking $A=$ min in order to apply it for the analysis of the parameters of the BLPP solving algorithm. Fuzzy sets $\mu_{1}, \mu_{2}, \ldots, \mu_{n}$ in this case are the membership functions of the lower level objectives, but fuzzy set $\mu_{0}$ is the membership function of the upper level objective:

$$
\begin{gathered}
\tilde{A}_{\mu_{0}}\left(\mu_{1}, \mu_{2}, \ldots, \mu_{n}\right)(x)= \\
=\max _{\mu_{0}(x)=\mu_{0}(u)} \min \left(\mu_{1}(u), \mu_{2}(u), \ldots, \mu_{n}(u)\right),
\end{gathered}
$$

where

$$
\mu_{1}, \ldots, \mu_{n} \in[0,1]^{D}, x, u \in D .
$$

Denoting $\tilde{A}_{\mu_{0}}\left(\mu_{1}, \mu_{2}, \ldots, \mu_{n}\right)(x)=\mu(x)$ we rewrite $\mu$ as $\mu(x)=z\left(y_{0}(x)\right)$, where $z$ is the function defined on the interval $\left[y_{0}^{\min }, y_{0}^{\max }\right]$ as follows: for $t \in\left[y_{0}^{\min }, y_{0}^{\max }\right]$ we take $x \in D$ such, that $t=y_{0}(x)$, and set $z(t)=\tilde{A}_{\mu_{0}}\left(\mu_{1}, \mu_{2}, \ldots, \mu_{n}\right)(x)$ (it is easy to see, that this value doesn't depend on $x)$.

Now we consider two functions $z_{0}$ (introduced in the second section) and $z$ (defined by the result of aggregation). The graphical analysis of these functions (i.e. the graphical analysis of two lines $\alpha=z-0(t)$ and $\alpha=z(t))$ helps us to choose parameters $\Delta_{L}, \Delta_{U}$ and $\delta$ correctly.

Let us consider the following example.

\section{Example 4.2}

$$
\begin{gathered}
P^{U}: \quad y_{0}(x)=x_{1}-x_{2} \longrightarrow \min \\
P_{1}^{L}: \quad y_{1}(x)=-0.2 x_{1}-x_{2} \longrightarrow \min \\
P_{2}^{L}: \quad y_{2}(x)=x_{2} \longrightarrow \min \\
D:\left\{\begin{array}{l}
x_{2} \leq 6, \\
5 x_{1}+x_{2} \leq 15 \\
x_{l} \geq 0, x_{2} \geq 0
\end{array}\right.
\end{gathered}
$$

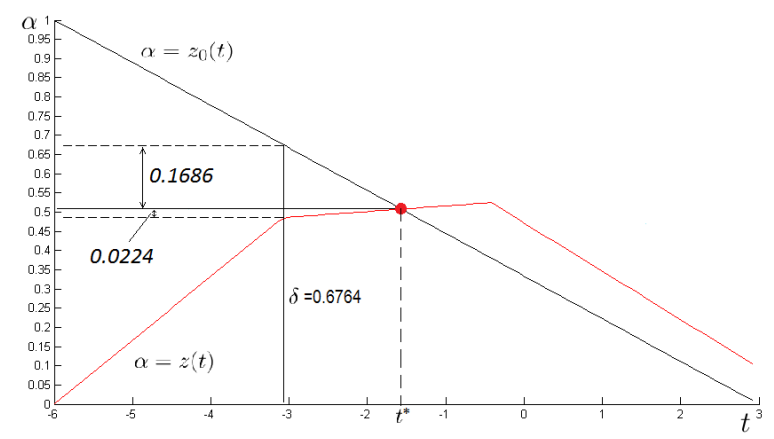

Figure 2: Analysis of solving parameters for Example 4.2

The graphical analysis of the parameters could be performed by Fig. 2. The intersection of lines $\alpha=z_{0}(t)$ and $\alpha=z(t)$ on Fig. 2 points out the optimal solution $x^{*}$ of the MOLP problem without any hierarchy between objectives: $t^{*}=y_{0}\left(x^{*}\right)$. In our case we are dealing with BLPP, when objective function $y_{0}$ is minimized with the higher priority than objectives $y_{1}$ and $y_{2}$. The compromised solution $x^{*}$ gives us the degree of satisfaction of the upper level objective $\delta=0.51$. But the analysis of Fig. 2 allows us to see, that a minor decrease for 0.0224 in the degree of minimization on the lower level, which is characterized by the result of factoraggregation $z(t)$, will give us a significant increase for 0.1686 in the degree of minimization $\delta$ on the upper level. It means, that we would rather choose point $t^{* *}=-3.05$ to obtain the optimal solution $x^{* *}$ for the BLPP, than point $t^{*}$, which gives us the solution without priority for the upper level objective. The similar graphical analysis could be performed, when we first choose the values of parameters $\Delta_{L}$ and $\Delta_{U}$, which characterize the degree of minimization on the lower level, and then we can find out the 
possible values of the degree of minimization $\delta$ for the upper level objective.

We consider some properties of function $z$, which describes the output value of factoraggregation of the lower level objectives. Function $z_{0}$ used for representation of the upper level objective membership function is decreasing on interval $\left[y_{0}^{\min }, y_{0}^{\max }\right]$. Now we consider properties of function $z$ on interval $\left[y_{0}^{\min }, y_{0}^{\max }\right]$. Let us denote $t^{*}=y_{0}\left(x^{*}\right)$, where $\left(x^{*}, \sigma^{*}\right)$ is the solution of MOLP without any hierarchy between objectives.

\section{Proposition 4.3}

$$
\sigma^{*}=\min \left\{z\left(t^{*}\right), z_{0}\left(t^{*}\right)\right\} .
$$

Proof. We recall that

$$
\sigma^{*}=\min \left\{\mu_{0}\left(x^{*}\right), \ldots, \mu_{n}\left(x^{*}\right)\right\}
$$

and consider two cases.

1) If $\sigma^{*}=\mu_{0}\left(x^{*}\right)$, then on the one hand

$$
\sigma^{*}=z_{0}\left(t^{*}\right)
$$

but on the other hand

$$
\begin{gathered}
z_{0}\left(t^{*}\right)=\mu_{0}\left(x^{*}\right) \leq \min \left\{\mu_{1}\left(x^{*}\right), \ldots, \mu_{n}\left(x^{*}\right)\right\} \leq \\
\leq \max _{\mu_{0}\left(x^{*}\right)=\mu_{0}(u)} \min \left\{\mu_{1}(u), \ldots, \mu_{n}(u)\right\}= \\
=\mu\left(x^{*}\right)=z\left(t^{*}\right),
\end{gathered}
$$

which means

$$
z_{0}\left(t^{*}\right)=\min \left\{z_{0}\left(t^{*}\right), z\left(t^{*}\right)\right\}
$$

2) Now let us suppose

$$
\sigma^{*}=\min \left\{\mu_{1}\left(x^{*}\right), \ldots, \mu_{n}\left(x^{*}\right)\right\}<\mu_{0}\left(x^{*}\right)
$$

and show that

$$
\sigma^{*}=\mu\left(x^{*}\right)=z\left(t^{*}\right)<z_{0}\left(t^{*}\right) .
$$

Considering

$$
\begin{gathered}
\sigma^{*}<\mu\left(x^{*}\right)= \\
=\max _{\mu_{0}\left(x^{*}\right)=\mu_{0}(u)} \min \left\{\mu_{1}(u), \ldots, \mu_{n}(u)\right\}
\end{gathered}
$$

and taking into account that $\sigma^{*}<\mu_{0}\left(x^{*}\right)$, we got the contradiction since $\sigma^{*}$ is the solution of MOLP.

\section{Proposition 4.4}

$$
\max _{t \in\left[y_{0}^{m i n}, t^{*}\right]} z(t)=z\left(t^{*}\right)
$$

Proof. Let us suppose that there exists

$$
\tau \in\left[y_{0}^{\min }, t^{*}\left[\text { such that } z(\tau)>z\left(t^{*}\right) \geq \sigma^{*} .\right.\right.
$$

Then we can find $u^{*} \in D$ such that

$$
\begin{aligned}
z(\tau) & =\max _{z_{0}(\tau)=\mu_{0}(u)} \min \left\{\mu_{1}(u), \ldots, \mu_{n}(u)\right\}= \\
& =\min \left\{\mu_{1}\left(u^{*}\right), \ldots, \mu_{n}\left(u^{*}\right)\right\}>\sigma^{*}
\end{aligned}
$$

and $\mu_{0}\left(u^{*}\right)=z_{0}(\tau)>\sigma^{*}$. As a result we got the contradiction since $\sigma^{*}$ is the solution of MOLP.

Proposition 4.5 Function $z$ is concave (convex upwards) on interval $\left[y_{0}^{\min }, y_{0}^{\max }\right]$.

Proof. We have to prove that

$$
z\left(\lambda t^{1}+(1-\lambda) t^{2}\right) \geq \lambda z\left(t^{1}\right)+(1-\lambda) z\left(t^{2}\right)
$$

for all $t^{1}, t^{2} \in\left[y_{0}^{\min }, y_{0}^{\max }\right]$ and for all $\lambda \in[0,1]$. Let us take such $x^{1}$ and $x^{2}$ that

$$
\begin{gathered}
t^{1}=y_{0}\left(x^{1}\right), t^{2}=y_{0}\left(x^{2}\right), \\
z\left(t^{1}\right)=\min \left\{\mu_{1}\left(x^{1}\right), \ldots, \mu_{n}\left(x^{1}\right)\right\}
\end{gathered}
$$

and

$$
z\left(t^{2}\right)=\min \left\{\mu_{1}\left(x^{2}\right), \ldots, \mu_{n}\left(x^{2}\right)\right\} .
$$

Then

$$
\begin{gathered}
\mu_{i}\left(\lambda x^{1}+(1-\lambda) x^{2}\right)=\lambda \mu_{i}\left(x^{1}\right)+(1-\lambda) \mu_{i}\left(x^{2}\right) \geq \\
\geq \lambda \min \left\{\mu_{1}\left(x^{1}\right), \ldots, \mu_{n}\left(x^{1}\right)\right\}+ \\
+(1-\lambda) \min \left\{\mu_{1}\left(x^{2}\right), \ldots, \mu_{n}\left(x^{2}\right)\right\}= \\
=\lambda z\left(t^{1}\right)+(1-\lambda) z\left(t^{2}\right)
\end{gathered}
$$

for all $i=1,2, \ldots, n$. Therefore

$$
\begin{gathered}
z\left(\lambda t^{1}+(1-\lambda) t^{2}\right)= \\
=\max _{\mu_{0}(u)=z_{0}\left(\lambda t^{1}+(1-\lambda) t^{2}\right)} \min \left\{\mu_{1}(u), \ldots, \mu_{n}(u)\right\} \geq \\
\geq \min \left\{\mu_{1}\left(\lambda x^{1}+(1-\lambda) x^{2}\right), \ldots, \mu_{n}\left(\lambda x^{1}+(1-\lambda) x^{2}\right)\right\} \geq \\
\geq \lambda z\left(t^{1}\right)+(1-\lambda) z\left(t^{2}\right) .
\end{gathered}
$$

Let us illustrate these properties with two examples.

\section{Example 4.6}

$$
\begin{gathered}
P^{U}: \quad y_{0}(x)=-3 x_{1}-x_{2} \longrightarrow \min \\
P_{1}^{L}: \quad y_{1}(x)=3 x_{1}-2 x_{2} \longrightarrow \min \\
P_{2}^{L}: \quad y_{2}(x)=x_{1}-x_{2} \longrightarrow \min \\
D:\left\{\begin{array}{l}
-x_{1}+3 x_{2} \leq 21, \\
x_{1}+3 x_{2} \leq 27, \\
4 x_{1}+3 x_{2} \leq 45, \\
3 x_{1}+x_{2} \leq 30 \\
x_{l} \geq 0, \quad x_{2} \geq 0
\end{array}\right.
\end{gathered}
$$




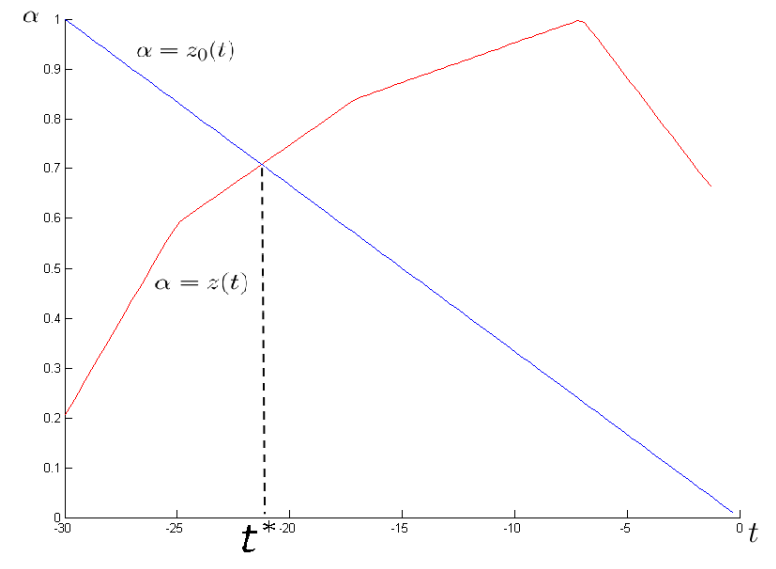

Figure 3: Lines $\alpha=z_{0}(t)$ and $\alpha=z(t)$ for Example 4.6

Line $\alpha=z(t)$ on Fig. 3 is concave and monotony increasing till the intersection point $t^{*}$, where the compromise solution could be found.

\section{Example 4.7}

$$
\begin{gathered}
P^{U}: \quad y_{0}(x)=x_{1}-x_{2} \longrightarrow \min \\
P_{1}^{L}: \quad y_{1}(x)=3 x_{1}-2 x_{2} \longrightarrow \min \\
P_{2}^{L}: \quad y_{2}(x)=-3 x_{1}-x_{2} \longrightarrow \min \\
D:\left\{\begin{array}{l}
-x_{1}+3 x_{2} \leq 21, \\
x_{1}+3 x_{2} \leq 27, \\
4 x_{1}+3 x_{2} \leq 45, \\
3 x_{1}+x_{2} \leq 30, \\
x_{l} \geq 0, x_{2} \geq 0
\end{array}\right.
\end{gathered}
$$

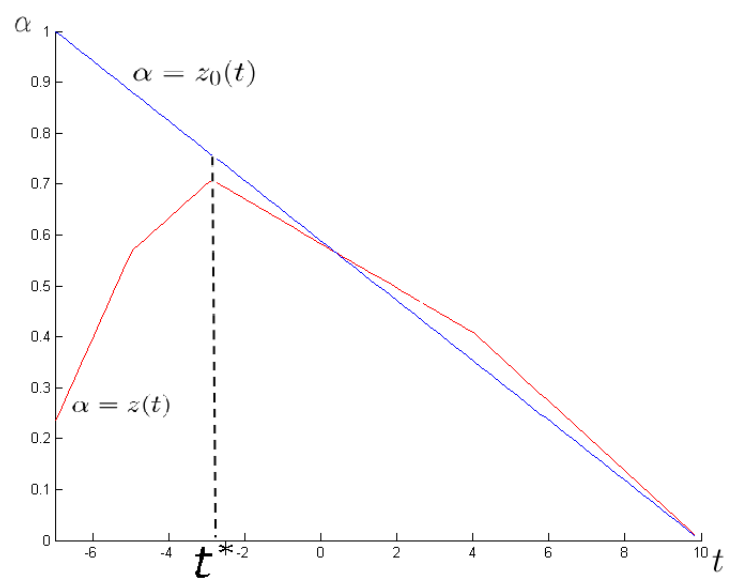

Figure 4: Lines $\alpha=z_{0}(t)$ and $\alpha=z(t)$ for Example 4.7

Line $\alpha=z(t)$ on Fig. 4 is concave too, but the compromise solution now could be found in point $t^{*}$, which is not the intersection of lines $\alpha=z(t)$ and $\alpha=z_{0}(t)$. And line $\alpha=z(t)$ is monotony increasing till this point.

\section{Mixed production planning problem}

We consider the following modification of the mixed production planning problem described by J.C. Figueroa-Garcia et al. in [3]. The goal of the mixed production planning problem is to determine the most profitable manufacturing plan at the same time minimizing environmentally dangerous products and the dependence on the outsource companies:

$$
\begin{gathered}
P^{U}: \sum_{j \in \mathbb{N}_{J}} \sum_{i \in \mathbb{N}_{I}} s p_{j i}\left(x_{j i}^{r}+x_{j i}^{o}+x_{j i}^{s}\right)- \\
-\left(c p_{j i}^{r} x_{j i}^{r}+c p_{j i}^{o} x_{j i}^{o}+c p_{j i}^{s} x_{j i}^{s}\right) \longrightarrow \max \\
P_{1}^{L}: \sum_{j \in \mathbb{N}_{J}} \sum_{i \in \mathbb{N}_{I}} e c_{j i}\left(x_{j i}^{r}+x_{j i}^{o}+x_{j i}^{s}\right) \longrightarrow \min \\
P_{2}^{L}: \sum_{j \in \mathbb{N}_{J}} \sum_{i \in \mathbb{N}_{I}} w_{j i} x_{j i}^{s} \longrightarrow \min
\end{gathered}
$$

$\sum_{i \in \mathbb{N}_{R}} \sum_{j \in \mathbb{N}_{J}} r m_{j i r}\left(x_{j i}^{r}+x_{j i}^{o}\right) \leq a m_{i r}, \quad i \in \mathbb{N}_{I}, \quad r \in \mathbb{N}_{R}$,

$$
\begin{aligned}
& x_{j i}^{r}, x_{j i}^{o}, x_{j i}^{s} \geq 0, \quad x_{j i}^{s} \leq a s_{j i}, \quad j \in \mathbb{N}_{J}, \quad i \in \mathbb{N}_{I}, \\
& d_{j i}^{(-)} \leq x_{j i}^{r}+x_{j i}^{o}+x_{j i}^{s} \leq d_{j i}^{(+)}, \quad j \in \mathbb{N}_{J}, \quad i \in \mathbb{N}_{I} .
\end{aligned}
$$

Index sets:

set $\mathbb{N}_{R}=\{1,2, \ldots, R\}$ of all resources $r \in \mathbb{N}_{R}$, set $\mathbb{N}_{J}=\{1,2, \ldots, J\}$ of all products $j \in \mathbb{N}_{J}$, set $\mathbb{N}_{I}=\{1,2, \ldots, I\}$ of all periods $i \in \mathbb{N}_{I}$.

Decision variables:

$x_{j i}^{r}$ - quantity of product $j$ to be manufactured in regular time in the period $i$,

$x_{j i}^{o}$ - quantity of product $j$ to be manufactured in overtime in the period $i$,

$x_{j i}^{s}$ - quantity of product $j$ to be manufactured by outsourcing in the period $i$.

Parameters:

$S P=\left(s p_{j i} \mid j \in \mathbb{N}_{J}, i \in \mathbb{N}_{I}\right)$, where $s p_{j i}$ is a sell price of product $j$ in the period $i$,

$C P^{r}=\left(c p_{j i}^{r} \mid j \in \mathbb{N}_{J}, i \in \mathbb{N}_{I}\right)$, where $c p_{j i}^{r}$ is a product $j$ production cost in the period $i$ for regular time,

$C P^{o}=\left(c p_{j i}^{o} \mid j \in \mathbb{N}_{J}, i \in \mathbb{N}_{I}\right)$, where $c p_{j i}^{o}$ is a product $j$ production cost in the period $i$ for overtime, $C P^{s}=\left(c p_{j i}^{s} \mid j \in \mathbb{N}_{J}, i \in \mathbb{N}_{I}\right)$, where $c p_{j i}^{s}$ is a product $j$ production cost in the period $i$ for outsourcing, $E C=\left(e c_{j i} \mid j \in \mathbb{N}_{J}, i \in \mathbb{N}_{I}\right)$, where $e c_{j i}$ is an evaluation of damage to environment caused by product $j$ in the period $i$,

$W=\left(w_{j i} \mid j \in \mathbb{N}_{J}, i \in \mathbb{N}_{I}\right)$, where $w_{j i}$ is a weight of outsource product $j$ in the period $i$, $R M=\left(r m_{j i r} \mid j \in \mathbb{N}_{J}, i \in \mathbb{N}_{I}, r \in \mathbb{N}_{R}\right)$, where $r m_{j i r}$ is an amount of the $r$ raw material units used to manufacture product $j$ in the period $i$,

$A M=\left(a m_{i r} \mid r \in \mathbb{N}_{R}, i \in \mathbb{N}_{I}\right)$, where $a m_{i r}$ is an availability of the raw material type $r$ in the pe$\operatorname{riod} i$ 
$A S=\left(a s_{j i} \mid j \in \mathbb{N}_{J}, i \in \mathbb{N}_{I}\right)$, where $a s_{j i}$ is a number of available outsourced units of product $j$ in the period $i$,

$D^{-}=\left(d_{j i}^{(-)} \mid j \in \mathbb{N}_{J}, i \in \mathbb{N}_{I}\right)$, where $d_{j i}^{(-)}$is a minimum demand of product $j$ in the period $i$,

$D^{+}=\left(d_{j i}^{(+)} \mid j \in \mathbb{N}_{J}, i \in \mathbb{N}_{I}\right)$, where $d_{j i}^{(+)}$is a maximum (potential) demand of product $j$ in the period $i$.

Maximization problem $P^{U}$ is reduced to the minimization problem by taking the profit function with the minus sign. This numerical example uses the following values of the parameters:

$I=1, J=10, R=5$,

$A M=\left(9 \cdot 10^{6}, 4 \cdot 10^{6}, 4.5 \cdot 10^{6}, 3 \cdot 10^{6}, 5.5 \cdot 10^{6}\right)$,

$E C=(8,9,5,4,3,7,2,1,6,10)$.

Table 1 contains the values of $R M$. The values of other parameters are given by Table 2 .

\begin{tabular}{|c|c|c|c|c|c|}
\hline$r$ & 1 & 2 & 3 & 4 & 5 \\
\hline 1 & 50.47 & 83.37 & 90.29 & 133.27 & 71.75 \\
\hline 2 & 53.46 & 79.93 & 84.88 & 133.87 & 55.69 \\
\hline 3 & 106.49 & 75.30 & 101.81 & 113.06 & 96.03 \\
\hline 4 & 125.26 & 103.13 & 94.35 & 59.82 & 134.97 \\
\hline 5 & 93.96 & 120.50 & 100.36 & 134.71 & 78.87 \\
\hline 6 & 137.24 & 87.68 & 40.55 & 110.17 & $\begin{array}{l}93.26 \\
\end{array}$ \\
\hline 7 & 136.14 & 112.83 & 67.93 & 96.40 & 77.01 \\
\hline 8 & 72.47 & 53.75 & 124.05 & 110.74 & 99.43 \\
\hline 9 & 56.53 & 42.53 & 44.42 & 66.05 & $\begin{array}{l}97.82 \\
\end{array}$ \\
\hline 10 & 98.72 & 109.48 & 56.77 & 103.07 & 95.72 \\
\hline
\end{tabular}

Table 1: Values of parameters $r m_{j r}$ for the mixed production planning problem

\begin{tabular}{|c|l|l|l|c|l|l|l|}
\hline$j$ & $c p_{j}^{r}$ & $c p_{j}^{s}$ & $s p_{j}$ & $a s_{j}$ & $d_{j}^{(+)}$ & $d_{j}^{(-)}$ & $w_{j}$ \\
\hline 1 & 255 & 260 & 350 & 1237 & 8775 & 3900 & 0.07 \\
\hline 2 & 165 & 200 & 300 & 1107 & 7650 & 3400 & 0.15 \\
\hline 3 & 160 & 185 & 280 & 1519 & 6075 & 2700 & 0.07 \\
\hline 4 & 105 & 130 & 210 & 2636 & 7875 & 1500 & 0.05 \\
\hline 5 & 205 & 240 & 300 & 1979 & 6300 & 3400 & 0.05 \\
\hline 6 & 175 & 190 & 305 & 1617 & 7650 & 4000 & 0.08 \\
\hline 7 & 160 & 210 & 270 & 1442 & 5000 & 1500 & 0.05 \\
\hline 8 & 225 & 245 & 315 & 1527 & 6300 & 4000 & 0.05 \\
\hline 9 & 53 & 105 & 190 & 2266 & 4725 & 1000 & 0.18 \\
\hline 10 & 74 & 120 & 220 & 2500 & 8775 & 2600 & 0.25 \\
\hline
\end{tabular}

Table 2: Values of parameters for the mixed production planning problem

The graphical analysis of lines $\alpha=z_{0}(t)$ and $\alpha=z(t)$ is given by Fig.5. The intersection of lines $\alpha=z_{0}(t)$ and $\alpha=z(t)$ points out the optimal solution $x^{*}$ of MOLP problem without any hierarchy between objectives: $t^{*}=y_{0}\left(x^{*}\right)$. By setting $\Delta_{L}=0.7$ and $\Delta_{U}=0.8$ we can observe that $\delta$ should lie in interval $[0.765,0.799]$, otherwise if $\delta>0.799$ then a solution does not exist. The graphical analysis shows that as the optimal solution of the mixed production planning problem it is natural to take $x^{* *}$

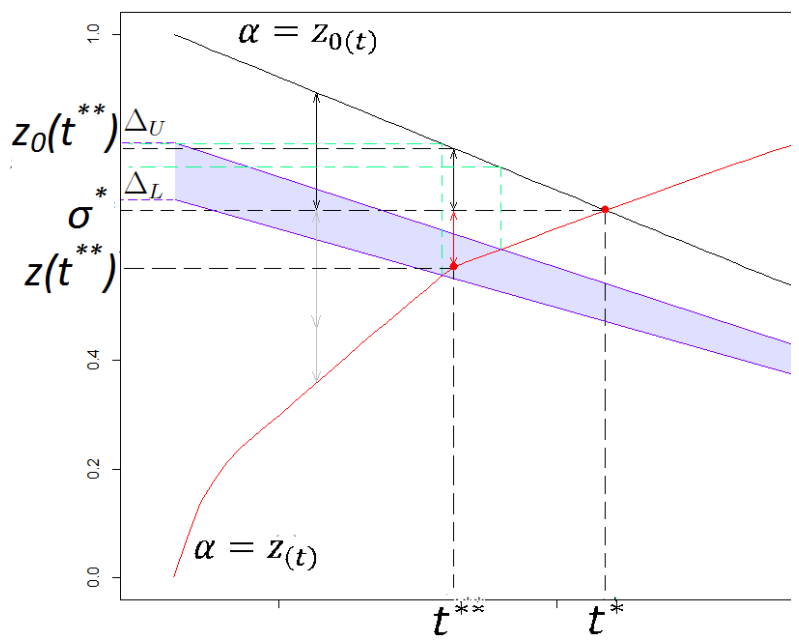

Figure 5: Analysis of solving parameters for the mixed production planning problem

such that $t^{* *}=y_{0}\left(x^{* *}\right)$.

\section{Conclusions}

In this paper we introduce the construction of factoraggregation, which is a case of the general aggregation operator. This construction is investigated and applied for the analysis of parameters of the bilevel linear programming solving algorithm. The numerical example illustrates how factoraggregation helps us to choose solving parameters accordingly to our vision of the hierarchy between objective functions.

The considered application uses factoraggregation defined by means of minimum as the initial aggregation. Our future work will focus on investigation and comparison of factoraggregation operators based on different initial operators.

\section{Acknowledgement}

This work has been supported by the European Social Fund within the project "Support for Doctoral Studies at University of Latvia" and within the project "Support for Master Studies at University of Latvia".

\section{References}

[1] T. Calvo, G. Mayor and R. Mesiar. Aggregation Operators, Physical-Verlag, Heidelberg, 2002.

[2] M. Detyniecki. Fundamentals on Aggregation Operators, Berkeley, 2001.

[3] J.C. Figueroa-Garcia, D. Kalenatic and C.A. Lopez-Bello, Multi-period mixed production planning with uncertain demands: fuzzy and interval fuzzy sets approach, Fuzzy Sets and Systems, 206:21-38, Elsevier, 2012.

[4] M. Grabisch, J.-L. Marichal, R. Mesiar and E. Pap. Aggregation Functions, Cambridge University Press, 2009. 
[5] M. Jiménez and A. Bilbao, Pareto-optimal solutions in fuzzy multi-objective linear programming, Fuzzy Sets and Systems, 160:2714-2721, Elsevier, 2009.

[6] E.P. Klement, R. Mesiar and E. Pap. Triangular Norms, Kluwer Academic Publishers, Dordrecht, 2000.

[7] M. Sakawa and I. Nishizaki, Interactive fuzzy programming for decentralized two-level linear programming problems, Fuzzy Sets and Systems, 125:301-315, Elsevier, 2002.

[8] M. Sakawa, I. Nishizaki and Y. Uemura, Interactive fuzzy programming for multi-level linear programming problems, Comput. Math. Appl., 36:71-86, 1998.

[9] A. Takaci, General aggregation operators acting on fuzzy numbers induced by ordinary aggregation operators, Novi Sad J. Math, 33(2):67-76, 2003.

[10] H.J. Zimmermann, Fuzzy programming and linear programming with several objective functions, Fuzzy Sets and Systems, 1:45-55, Elsevier, 1978. 